\title{
A Universal U-model based Control System Design
}

\author{
ZHU Quanmin ${ }^{1,3}$, LI Shaoyuan ${ }^{2}$, ZHAO Dongya ${ }^{3}$ \\ 1. Department of Engineering Design and Mathematics, University of the West of England, Frenchay Campus, Coldharbour Lane, Bristol, \\ BS16 1QY, UK \\ E-mail: quan.zhu@uwe.ac.uk
}

2. Department of Automation, Shanghai Jiao Tong University, Shanghai, China, 200240

E-mail: $\underline{\text { syli@ } @ \text { sjtu.edu.cn }}$

3. College of Chemical Engineering, China University of Petroleum, Qingdao, China, 266580 dyzhao@upc.edu.cn

\begin{abstract}
In conjunction with some of the latest research results, this study briefly summarizes the major characteristics with U-model based control system design and foresees the potential blue-sky research issues. The purpose of the presentation is to promote the awareness of the research methodology and to collect critical comments on the shortcomings with the less known approaches, which could stimulate further research and applications.
\end{abstract}

Key Words: U-model, U-block model, pole placement control, linearization, state space framework, linear and nonlinear dynamics

\section{Introduction}

So far almost all control system design approaches, no matter if they are linear or nonlinear plant based, have taken a unique procedure, set up a specified control system performance/index and then by inverting the integrated function of the plant and design performance in a specifically structured closed loop framework, obtain the controller output (that is the input to the plant). There is no problem at all with such procedures for all linear plant based control system design subject to stability considerations, because the linear superposition principle makes the inverse function easily resolved. However when the plants are subject to nonlinear dynamic equations (particularly those non-affine pure feedback general nonlinear system models [1]), the inverse functions are more complicated, intractable, or even impossible with analytical solutions, except step by step numerical computations or neural network based inductive approaches [2]. To further study the above challenging problems, a few of research questions are asked to provide possible solution platforms.

Research question one: Can a class of nonlinear dynamic plants, described by smooth nonlinear models in terms of polynomials and state space descriptions, be designed directly using the approaches developed from linear control systems?

Research question two: Is there any general control oriented model framework available to implement the linear approaches based control system design?

Research question three: Is there any general approach to resolve the inverse of nonlinear functions/models?

\footnotetext{
* This work is partially supported by the National Nature Science Foundation of China under Grant 61004080, 61273188, Shandong Provincial Natural Science Foundation under Grant ZR2011FM003, China and the Fundamental Research Funds for the Central Universities of China, Development of key technologies project of Qingdao Economic and Technological Development Zone under Grant 2011-2-52, Taishan Scholar Construction Engineering Special Funding.
}

Research question four: If such ad hoc design methodologies exist for nonlinear dynamic models. How will these be applied in designing the control systems with classical linear dynamic models?

With such insight, the U-model methodology has been proposed, where the origin, but not in general U-model expression, was appeared in one of the authors $\mathrm{PhD}$ thesis [3]. In progression along this route, The first time of the U-model was named in a study of pole placement controller design for nonlinear plants [4], which is a simple mapping from ordinary linear and nonlinear difference equations to time-varying polynomials in terms of the plant input $\mathrm{u}(\mathrm{t}-1)$ (that is the controller output). The U-model covers almost all existing smooth nonlinear discrete time polynomial models as its subsets. A summary of the first decade research development has been reported in this research field [5]. It should be noted that the U-model associated publications are still in a very beginning stage. Although various algorithms have been developed and bench tested with simulations and applications, they have not had rigorous analytical description and top journal publication till today.

The rest of the studies are organized from model structure formulation/properties (Section 2), control design framework/procedure (Section 3), to summary of the paper (Section 4).

\section{U-model --- control oriented model}

Consider a general nonlinear polynomial plant model below.

$$
\begin{aligned}
& y(t)=f\left(y(t-1), \ldots, y\left(t-n_{y}\right), u(t-1), \ldots, u\left(t-n_{u}\right)\right) \\
& y(t)=\sum_{l=0}^{L} p_{l}(t) \theta_{l}
\end{aligned}
$$

where $y(t) \in R$ and $u(t) \in R$ are the output and input (also known as controller output in control system design) of the plant respectively at discrete time instant $t(1,2, \ldots)$, the 
regression terms $p_{l}(t)$ are the products of past inputs and outputs such as $u(t-1) y(t-3), u(t-1) u(t-2), y^{2}(t-1)$, and $\theta_{l}$ are the associated parameters. Typically, for example, linear time invariant difference equation based plant models and NARMA (nonlinear auto-regressive moving average) models [10].

The corresponding U-model is defined as below

$$
y(t)=\sum_{j=0}^{M} \lambda_{j}(t) u^{j}(t-1)
$$

This is expanded from the above nonlinear function $f($.) as a polynomial with respect to $u(t-1)$. where $M$ is the degree of model input (controller output) $u(t-1)$, the time varying parameter vector $\lambda(t)=\left[\begin{array}{lll}\lambda_{0}(t) & \cdots & \lambda_{M}(t)\end{array}\right] \in R^{M+1}$ is a function of past inputs, outputs $(u(t-2), \ldots, u(t-n), y(t-1), \ldots$, $y(t-n))$, and the parameters $\left(\begin{array}{lll}\theta_{0} & \cdots & \theta_{L}\end{array}\right)$ in (2.1).

\subsection{Transform properties}

Let $\varphi: R^{L+1} \rightarrow R^{M+1}$ be the map from polynomial model to U-model and its inverse be $\varphi^{-1}$, that is

$$
f(P(*), \Theta) \stackrel{\varphi}{\longrightarrow} f(\Upsilon(*), \mathrm{U}(t-1))
$$

Then it has the following properties [7].

- The map is injective (one to one)

- The map is surjective (onto)

- Therefore the map is bijective as it is both injective and surjective.

- The map is invertible.

- The map does not change any both model characteristics, such as output response, stability, dynamics and statics.

Within the U-model framework, there is nothing in change of the polynomial model properties such as dynamic, stability, and input and output external relationship. However by such mapping (re-organization) of the model expression, U-model provides a generic prototype to facilitate control system design by using linear approaches.

\subsection{U equivalent state space models}

Problem formulation: for a given a nonlinear polynomial model, how can the state space model based design approaches, particularly those linear state space model based design approaches be applied? In fact this brings forward a scheme to make nonlinear polynomial models in state space realization.

Justification: in practice, polynomial models are frequently obtained from principle and/or data driven identification, particularly in the cases of single-input and single-output (SISO) dynamic plants, even though maybe with complex dynamics and higher nonlinear degrees. On the other hand, state space based control system design is much more concise and convenient in mathematical manipulation, especially, Lyapunov stability analysis based design approaches. Except U-model based approach, there has been no other general realization procedure to transform a nonlinear polynomial model into its proper state space expression. It should be mentioned that input-state linearization and input-output linearization techniques are those to process nonlinear state space models into equivalent liner expression by coordinate transform [8].

Procedure/properties: The realization of a nonlinear polynomial model to its $U$ equivalent state space model has been initially studied [7], which the step by step procedure is listed below

- With reference to polynomial U-model, assign/design a closed loop system with specified poles and zero steady error. Consequently the input-output relationship is linearized with the closed loop system. The linearized model is defined as U-Block model.

- Subsequently by classical approaches (such as controllable or observable realization) convert the U-Block model into its state space realization.

\subsection{U state space models}

Consider a general discrete time state space model description below, which includes all the currently studied combinational models of affine, non-affine, strict feedback, and pure feedback descriptions as its subsets.

$$
\begin{aligned}
& x(t+1)=f(x(t), \Upsilon(t), u(t)) \\
& =f_{x}(x(t), \Upsilon(t))+f_{u}(x(t), \Upsilon(t), u(t)) \\
& y(t)=h(x(t))
\end{aligned}
$$

where $x(t) \in R^{n}, \quad u(t) \in R, \quad y(t) \in R$ are the state variable, system input and output respectively. $f_{x}(x(t), \Upsilon(t))$ and $f_{u}(x(t), \Upsilon(t), u(t))$ are the summations of the products formed with $(x(t), \Upsilon(t))$ and $(x(t), \Upsilon(t), u(t))$ respectively. Further $\Upsilon(t)=\Upsilon(x(t), u(t-1), \ldots, u(t-n)) \in R^{m} \quad, \quad$ which excludes the current model input $u(t)$. Consequently the control oriented $\mathrm{U}$ state space model is defined as below

$$
\begin{aligned}
& x(t+1)=f(x(t), \Upsilon(t), u(t)) \\
& =f_{x}(x(t), \Upsilon(t))+U(t) \\
& y(t)=h(x(t))
\end{aligned}
$$

Example 1 control oriented total nonlinear state space model

$$
\begin{aligned}
& x_{1}(t+1)=0.6 x_{1}(t) x_{2}(t)+0.2 x_{2}(t) u^{3}(t)+u(t) u(t-1)+u^{2}(t) \\
& x_{2}(t+1)=0.3 x_{1}(t) u(t)+0.5 x_{2}(t)-0.2 x_{2}^{3}(t)+u(t) u(t-2) \\
& y(t)=x_{1}(t)+x_{2}(t)
\end{aligned}
$$

This comes from the fact that those models with input, state, and output are all subject to nonlinear and have effect to controller design.

Example 2 total nonlinear state space model

$$
\begin{aligned}
& x_{1}(t+1)=\frac{0.6 x_{1}(t) x_{2}(t)+0.2 x_{2}(t) u^{3}(t)+u(t) u(t-1)+u^{2}(t)}{1+0.2 x_{2}^{2}(t)+0.3 u^{2}(t)} \\
& x_{2}(t+1)=\frac{0.3 x_{1}(t) u(t)+0.5 x_{2}(t)-0.2 x_{2}^{3}(t)+u(t) u(t-2)}{1+0.3 x_{1}^{2}(t) x_{2}^{2}(t)+u^{2}(t)} \\
& y(t)=x_{1}(t)+x_{2}(t)
\end{aligned}
$$

This comes from the fact that those models with input, state, output plus parameters (this make sense of real 
nonlinear issues in parameter estimation/system identification) are ALL/TOTAL nonlinear.

Justification: It should be mentioned that the above two examples may not be realistic or no practical representations at the moment, even though can be mathematically expressed. Due to the generality of the model structure, any solution in control would be further generalized the methodologies on nonlinear control system design. Of course this brings more challenging research topics.

Procedure/properties: This is the first time to propose $\mathrm{U}$ state space model structure. The model properties have not been studied. It cannot find analysis and ideas on the model expression, mapping, operations, and so on from publically available literature. Particularly the authors have checked through a classical authoritative work [8].

\subsection{Section summary}

- The coefficients associated with the control input $u(t)$ in U-model are time varying because of absorbing the past inputs and outputs.

- The U-model structure is not only to facilitate nonlinear control system design, but also provide a new prototype in designing linear control systems, which will be explained in next section.

- Need further rigorous mathematical studies on the model structure and its properties. U-model has facilitated nonlinear control system design, is there any way to facilitate the stability analysis with the model structure?

- Can those classical results/properties on time varying models be introduced for the U-model structure study?

\section{Control system design}

Hypothesis: It is possible to use linear controller design methodologies to design a large of smoothly nonlinear plant based control systems, and therefore to simplify and generalise nonlinear control system design [7].

In order to use linear polynomial mode based design approaches, define the desired plant output as $U(t)$. With reference to U-model (2.2), in simple mathematical expression, it is clear to have

$$
y(t)=U(t)
$$

Then it gives

$$
U(t)=\sum_{j=0}^{M} \lambda_{j}(t) u^{j}(t-1)
$$

Accordingly the task of the design is to determine the desired plant output $U(t)$ according to a specified performance index, for example,

Pole Placement Control [4]

$$
R U(t)=T w(t)-S y(t)
$$

General Predictive Control [9]

$$
J_{p}=E\left[Y_{d}^{T} Q Y_{d}+\Delta U^{T} \lambda \Delta U\right]
$$

Then the remaining design task is to resolve one of the roots of (3.2) to obtain the controller output. That is

$$
u(t-1)=\Psi\left[U(t)-\sum_{j=0}^{M} \lambda_{j}(t) u^{j}(t-1)=0\right]
$$

where $\Psi[*]$ is a root-solving algorithm, such as Newton-Raphson algorithm. A detailed analysis on the root solving issues has been presented [4].

Justification: U-model based design procedure is different from classcial ones. U-model procedure is split into two steps, design plant output (instead of (plant input, i.e. controller output), then resolve the controller output from the designed/specified plant output. This can make once off desing and then be applied to many different plants [4,7]. A comparison is presented for classical design procedure against the U-model based design procedure for linear models in Apendix 1.

Properties: The other distinct property is the induced errors from U-model design procedure. With reference to (3.1) and (3.2), consider the errors in the design

$$
\begin{aligned}
& U(t)=\sum_{j=0}^{M} \lambda_{j}(t) u^{j}(t-1)+\Delta(t) \\
& \Delta(t)=\Delta_{f}(t)+\Delta_{r}(t)+\Delta_{o}(t)
\end{aligned}
$$

where $\Delta_{f}(t)$ is the error induced from $y(t)$ following $U(t)$ and $\Delta_{r}(t)$ is the error induced from root solving numerical algorithms, which are uniquely appeared in U-model based design, $\Delta_{o}(t)$ is the representative to all the other possible errors/disturbances studied in classical control system design. Although simulation experiments have demonstrated the first two errors converged with the studied examples, thorough theoretical analysis has not been presented.

The following sub-sections briefly explain the research results obtained from previus studies and foresee the relevant potential blue-sky research issues.

\subsection{Design with $\mathrm{U}$ polynomial plant models}

Problem formulation: For a given nonlinear polynomial model, how can it be designed with linear polynomial approaches? To answer this question, two new definitions are presented. Let a linear control system design criterion or performance index $J\left(C_{1}, \mathrm{C}_{2}, \ldots \mathrm{C}_{n}\right) \in R^{n} \quad$, where $C_{1}, \mathrm{C}_{2}, \ldots \mathrm{C}_{n}$ are the polynomials and/or parameters specifying the control system performance index. Further let the designed plant output $U(t) \in R$ and the plant input (i.e. controller output) $u(t) \in R$. A continuous map $\varphi_{\text {struc }}: R^{n} \rightarrow R$ makes $J(*) \rightarrow U(t)$ is said to be feasible for using linear control design approaches to design the control of nonlinear polynomial plants. The other continuous map $\varphi_{\text {root }}: R \rightarrow R$ makes $U(t) \rightarrow u(t-1)$ is said to be invertible to resolve the controller output.

Justification: polynomial models are widely and frequently used in applications, particularly in the cases of single-input and single-output (SISO) dynamic plants, even though maybe with complex dynamics and higher nonlinear degrees. As explained in previous section, even for linear polynomial models, U-model approach provides a new design methodology. This can be easily shown off with reference to (3.2), for linear models and affine nonlinear models (i.e. without higher power with plant input $u(t-1)$ ),

(3.2) becomes 


$$
U(t)=\lambda_{0}(t)+\lambda_{1}(t) u(t-1)
$$

Therefore it gives

$$
u(t-1)=\frac{U(t)-\lambda_{0}(t)}{\lambda_{1}(t)}
$$

Procedure/properties: The major property analyses have been conducted. In regarding to pole placement control, it has included the assignability for pole placement controller, the errors induced from following desired output and root solving algorithm. In regarding to general predictive control, it has included the boundness of U-model parameter set, comparison of mean squared error from using U-model and linearized model for reference to control nonlinear plants. It should be mentioned that the stability analysis has been yet presented. A future work could link U-model design approaches to the selection of PID (Proportional, Integral, and Derivative) controller's parameters.

\subsection{Design with $U$ equivalent state space plant models}

Problem formulation: How can a nonlinear polynomial model be designed by linear state space model based approaches?

Justification: State space model structure has been widely used for control system design. Lyapunov stability analysis can be efficiently applied to design various of control systems (such as sliding mode control) which cannot be easily designed by polynomial models.

Procedure/properties: The U-model based design procedure is shown in appendix. The $\mathrm{U}$ equivalent state space model structure has been used to design sliding model control systems [7]. Further studies could be the design of robust control systems with linear state space model based approaches and the design with Lyapunov stability analysis based approaches.

\subsection{Design with $U$ state space plant models}

Problem formulation: With reference to (2.4), design the requested control systems. There is no formulated procedure so far.

Justification: As described in section 2, state space model based designed approaches are dominant now days. While polynomial U-model and $\mathrm{U}$ equivalent state space model based design have been demonstrated workable (of course need further thorough studies). U state space model based design approaches should be studied as well as to the $\mathrm{U}$ polynomial model design methodology.

Procedure/properties: None at the moment. It is an open field.

\subsection{Section summary}

There are many topics need to be studied, list a few below

- Test all possible linear design approaches within the U-model framework.

- Compare classical approaches with U-model approaches for linear plant models.

- State space based approaches need to be comprehensively studied.

- As time varying nature of the U-model expression, the corresponding time varying stability analysis has not been touched.
- Need more rigorous mathematical studies on the model structure and design implications. Therefore it provides a thorough and systematic warrantee or wide range applications.

- A few of representative U-model based design schematic diagrams are shown for easily following the design flows in appendix 2.

\section{Conclusions}

Since the first official publication in 2002, U-model based control system design procedures have gone through a decade. It has not been a main research direction. Probably it will never be large cohort researchers attended domain for control system design. However its philosophy, merit, and formulation with <providing simple and general solutions to complicated problems $>$ have attraction to new theoretical development and application bench tests. The paper is presented to promote the awareness of the research methodology and potential applications, exchange of research results, networking colleagues in the subject.

\section{References}

[1] Wang, D. and Huang, J., Adaptive neural network control for a class of uncertain nonlinear systems in pure-feedback form, Automatica, Vol. 38, 1365-1372, 2002.

[2] Ge, S.Z., Li, G.Y., and Lee, T.H., Adaptive NN control for a class of strict-feedback discrete-time nonlinear systems, Automatica, Vol 39, 807-819, 2003.

[3] Zhu, Q.M., Identification and Control of nonlinear systems, $\mathrm{PhD}$ thesis, University of warwick, UK, 1989.

[4] Zhu, Q.M. and Guo, L.Z., A pole placement controller for nonlinear dynamic plants, Journal of Systems and Control Engineering, Proceedings of the Institution of Mechanical Engineers Part I, Vol. 216, No. 6, 467-476, 2002.

[5] Xu, F.X., Zhu, Q.M., Zhao, D.Y., and Li, S.Y., U-model based design methods for nonlinear control systems $-A$ survey of the development in the 1st decade, Control and Decision, Vol. 28, No. 7, 961-971, 2013 (in Chinese)

[6] Zhu, Q.M., Wang, Y.J,, Zhao, D.Y., Li, S.Y., and Billings, S.A., Review of rational (total) nonlinear dynamic system modelling, identification and control, Int. J. of Systems Science, 2014 (in press).

[7] Zhu, Q.M., Zhao, D.Y. and Zhang, J.H., A general U-Block model based procedure for using linear state space approaches to design control systems with smoothly nonlinear polynomial plant models --- plus a demo case of sliding mode control, 2013 (submitted).

[8] Isidori, A., Nonlinear control systems (3rd ed.), Berlin: Springer-Verlag, 1995.

[9] Du, W.X., Wu, X.L, and Zhu, Q.M., Direct design of U-model based generalized predictive controller (UMGPC) for a class of nonlinear (polynomial) dynamic plants, Proc. Instn. Mech. Enger, Part I: Journal of Systems and Control Engineering, V 226, 27-42, 2012.

[10] Billings, S.A., Nonlinear System Identification: NARMAX Methods in the Time, Frequency, and Spatio-Temporal Domains, Wiley, John \& Sons, Chichester, West Sussex, 2013.

Appendix 1 Comparison of classical design procedure and U-model based design procedure for linear models

Inspection of Figure 1, 
1) the classical control design formula can be easily derived as

$G_{\text {ctrl }}=\frac{G_{\text {closed }}}{\left(1-G_{\text {closed }}\right) G_{\text {plant }}}$

Obviously it cost extra design effort ( $G_{\text {closed }}$ is fixed) for different plant each time. Secondly to determine the controller output need the inverse of the combination of the plant transfer function and the specified closed loop transfer function.

2) the U-model based control design procedure can be formulated as

$$
\begin{aligned}
& U=G_{\text {ctrl }}(y, w) \\
& u=\left(G_{\text {plant }}\right)^{-1}
\end{aligned}
$$

As $U$ is determined once off, for different plant, the redesign is only to determine its inverse each time.

Remark: In regarding to linear control system design, although computational burden is not largely different, U-model based procedure provide a new insight and frame work. In regarding to nonlinear control system design, the explained computational burden is normally in large difference. U-model procedure significantly reduces the design complexity from the inverse of the functions and provides a crisp general solution.

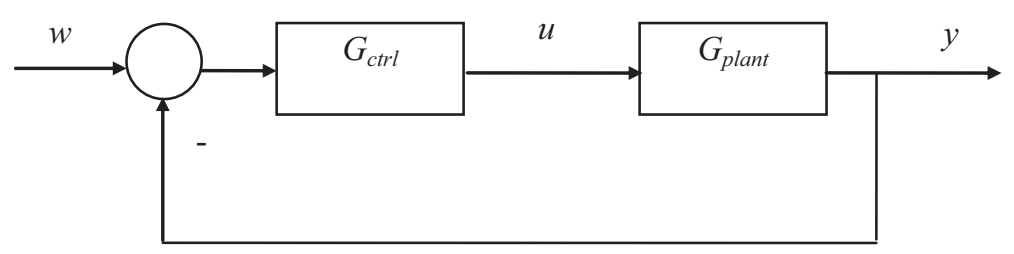

Figure 1(a) Classical linear control system structure

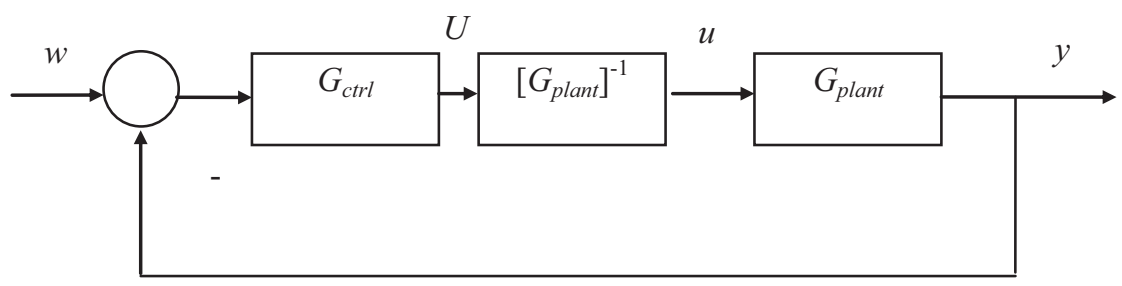

Figure 1(b) U-model based linear control system structure

Appendix 2 Block diagrams for U-model based control system structures and design flow chat

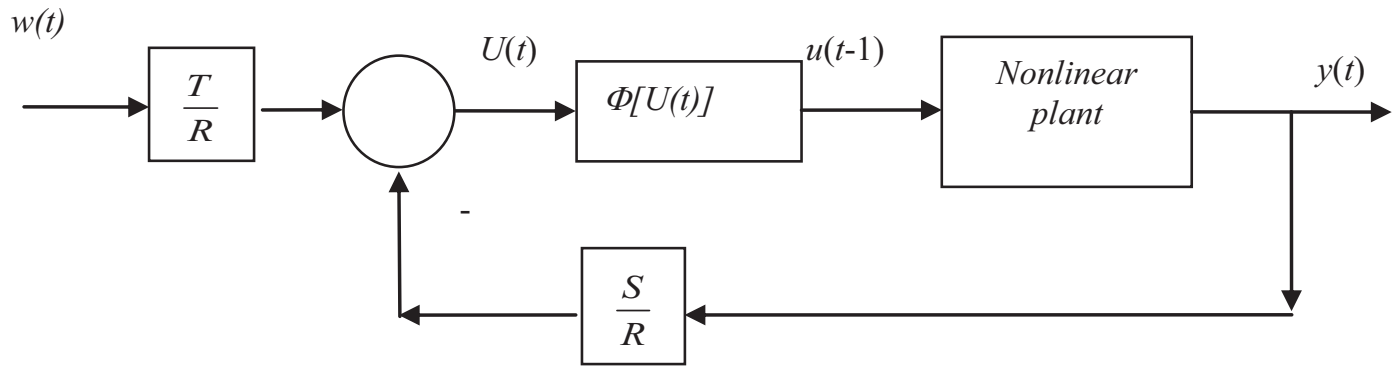

Figure 2 Diagram of U-model based pole placement control systems

$x(0)$

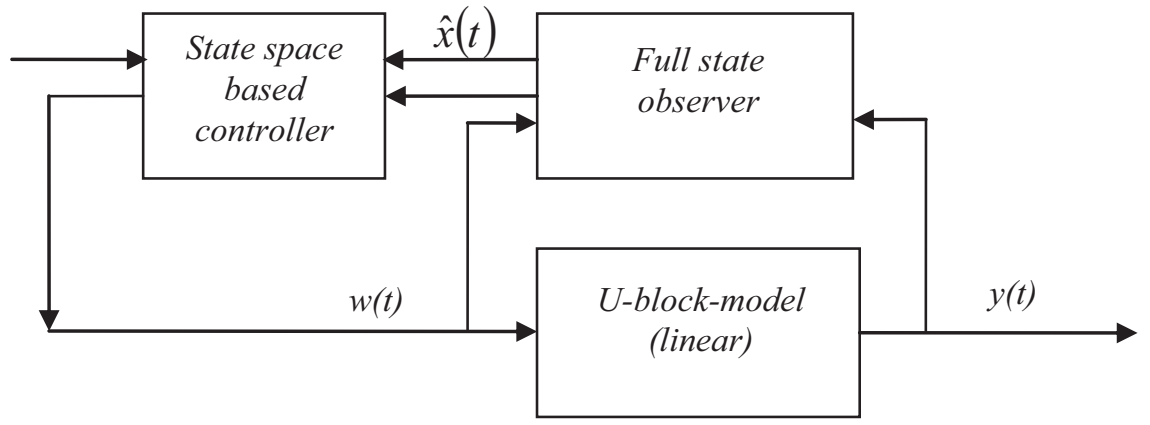

Figure 3 Diagram of U-block-model based control systems 


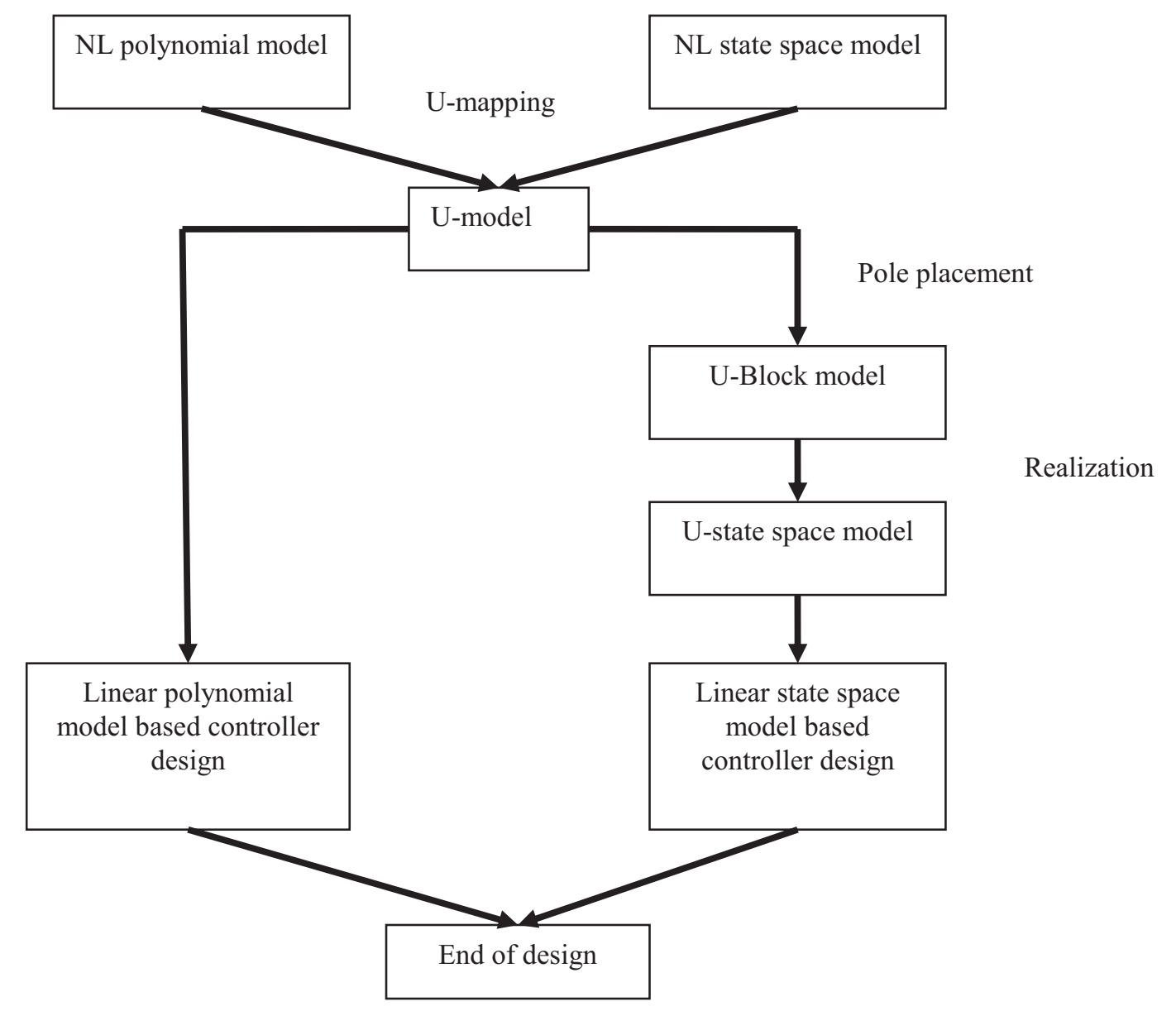

Figure 4 Flow chat of whole U-model based nonlinear control system design for both polynomial and state space models 